\title{
A Review of EEG Signal Analysis for Diagnosis of Neurological Disorders using Machine Learning
}

\author{
Vandana Joshi ${ }^{*}$ and Nirali Nanavati \\ Computer Engineering Department, Sarvajanik College of Engineering and Technology, Dr. R.K. Desai Marg, \\ Opp. Mission Hospital, Athwalines, Surat 395001, Gujarat, India
}

* e-mail: vandana.joshi@scet.ac.in

\begin{abstract}
Neurological disorders are diseases that affect the brain and the central autonomic nervous systems. These disorders take a huge toll on an individual's health and general well-being. After cardiovascular diseases, neurological disorders are the main cause of death. These disorders include epilepsy, Alzheimer's disease, dementia, cerebrovascular diseases including stroke, migraine, Parkinson's disease and numerous other disorders. This manuscript presents a state-of-the-art consolidated review of research on the diagnosis of the three most common neurological disorders using electroencephalogram (EEG) signals with machine learning techniques. The disorders discussed in this manuscript are the more prevalent disorders like epilepsy, Attention-deficit/hyperactivity disorder (ADHD), and Alzheimer's disease. This manuscript helps in understanding the details about EEG signal processing for diagnosis and analysis of neurological disorders along with a discussion of the datasets, limitations, results and research scope of the various techniques. (C) 2021 Journal of Biomedical Photonics \& Engineering.
\end{abstract}

Keywords: neurological disorders; electroencephalogram (EEG); epilepsy; attention-deficit/hyperactivity disorder (ADHD); Alzheimer.

Paper \#3435 received 2 Jun 2021; revised manuscript received 15 Jul 2020; accepted for publication 11 Aug 2021; published online 7 Sep 2021. doi: 10.18287/JBPE21.07.040201.

\section{Introduction}

It has been analyzed in various studies that neurological disorders are growing at a high rate. According to the WHO, at some point in their lives, one out of four people in the world would have neurological disorders [1]. Neurological disorders are the world's second most prevalent diseases, following ischemic heart disease $[1,2]$. Neurological disorders affect the brain as well as the nervous system of the human body [3].

The specific causes of the disorder can vary significantly depending upon the type of disorder as well as the specific area of the body that is affected. The causes may be due to genetic disorders, congenital abnormalities, infection, brain injury, etc. There are many known, relatively common neurological disorders but many are rare. Neurological disabilities include a wide range of disorders such as epilepsy, learning disabilities, neuromuscular disorders, autism, Alzheimer's disorder, ADHD, multiple sclerosis, Parkinson's disease, sleep disorder, and cerebral palsy.
On the other hand, mental disorders are "psychiatric diseases" or diseases that mainly appear as abnormalities in thought, feeling, or conduct, which lead to distress or function impairment [4].

Several brain-imaging tools are available for the diagnosis of these neurological disorders, including Positron Emission Tomography (PET), Near Infrared Spectroscopy (NIRS), Magnetoencephalogram (MEG), Electroencephalography (EEG), and Functional Magnetic Resonance Imaging (fMRI) [2]. This manuscript focuses on EEG analysis because it is a lowcost, non-invasive, portable technique, which is widely used. A standard electroencephalogram (EEG) measures and records the electrical activity of a case's brain to evaluate cerebral functions. There are numerous contributions in literature that analyze EEG signals for the detection of neurological disorders, early diagnosis of neuro-developmental disorders, detection of acute neurological events and monitoring the behavior of patients $[1,5]$. 
Numerous works in literature on EEG analysis have focused on epilepsy as one of the most common neurological disorders. Neurological disorders such as ADHD and Alzheimer's, however account for approximately one-third of the total diseases in developing nations. These disorders are often associated with an abnormal pattern of EEG and to analyze these EEG signals we need a well-trained neurologist. EEG signals are non-linear in nature. Some neuronal activity occurs because of minor changes in the voltage fluctuation of the EEG measures. The visual inspection of these signals, therefore, varies with the expertise. In addition, the long EEG recordings take a lot of time to review manually and the results sometimes cannot be exact and consistent [6]. This situation necessitates an automated system, which can be used, for neurological disorder detection and brain function monitoring with minimal human intervention [7]. This review will give an insight into the basics of EEG and how it is used to diagnose and analyze different neurological disorders.

The rest of the paper is organized as follows: in Section II and III, we discuss EEG principles and their processing phases respectively. Further, we discuss the investigation of three neurological disorders: Epilepsy in Section 4, ADHD in Section 5, and Alzheimer in Section 6 along with the results obtained by various works in literature. In Section 7, we discuss the problems and limitations for the detection of each disorder. The list of acronyms and abbreviations is summarized in Appendix A.

\section{EEG Overview}

In electroencephalogram (EEG), electrical currents are measured, generated by a group of specialized pyramidal cells within the brain which measure neuronal activity [6]. The electrical activity of a brain (EEG) exhibits significantly complex behavior with strong nonlinear and dynamic properties. Brain activity is measured by placing the electrodes on the scalp of the subjects. Positions of electrodes are defined by the 10-20 International Electrode Positioning System.

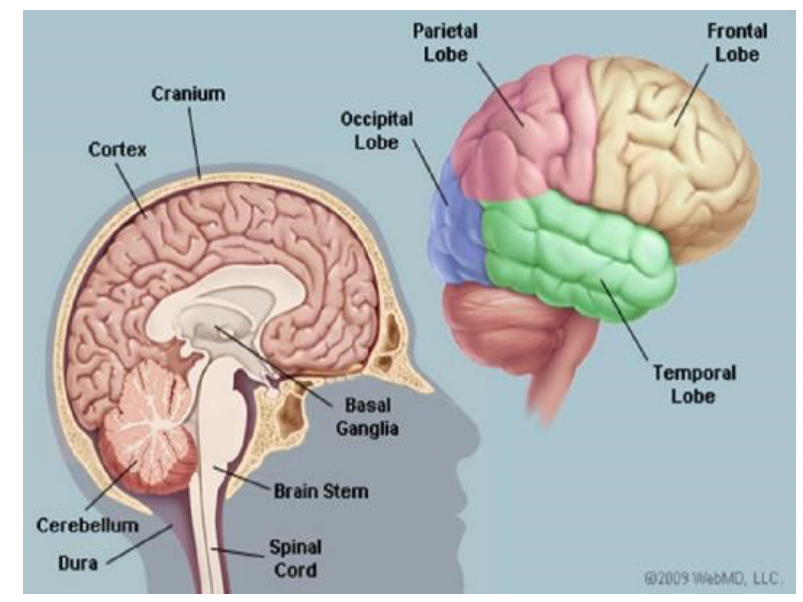

Fig. 1 Anatomical areas of the brain [8].
The brain is divided into three important parts anatomically: the cerebrum, cerebellum and brainstem, as illustrated in Fig. 1. The cerebrum contains two hemispheres: the right and left hemispheres. Each hemisphere is divided into four lobes: frontal, parietal, occipital and temporal. Each part is associated with brain functions related to thoughts, movements, emotions and motor functions. Table 1 illustrates the responsibility of each part.

Table 1 Anatomical region of brain associated with activity based on work [8].

\begin{tabular}{ll} 
Regions & Involve with / Responsible for \\
\hline Cerebral cortex & $\begin{array}{l}\text { Higher order cognitive tasks, such } \\
\text { as problem solving, language } \\
\text { comprehension, movement and } \\
\text { processing of complex visual } \\
\text { information }\end{array}$ \\
\hline Frontal lobe & $\begin{array}{l}\text { Personality, emotions, problem } \\
\text { solving, motor development, } \\
\text { reasoning, planning, parts of speech } \\
\text { and movement }\end{array}$ \\
\hline Parietal lobe & $\begin{array}{l}\text { Sensation (e.g., pain, touch), } \\
\text { sensory comprehension, } \\
\text { recognition, perception of stimuli, } \\
\text { orientation and movement }\end{array}$ \\
\hline Occipital lobe & Visual processing \\
\hline Temporal lobe & $\begin{array}{l}\text { Involved in dealing with the } \\
\text { recognition of auditory stimuli, } \\
\text { speech, perception and memory }\end{array}$ \\
\hline Cerebellum & $\begin{array}{l}\text { Motor control, sensory perception } \\
\text { and coordination, voluntary muscle } \\
\text { movements, fine motor skills, } \\
\text { posture and balance regulation }\end{array}$ \\
\hline
\end{tabular}

The brain signals are a combination of different base frequencies. These broad frequency ranges have been divided into several subgroups, called EEG rhythms or frequency bands. Each frequency band reflects a different state of mind or cognition in the brain (Table 2). Types of rhythms such as theta, delta, alpha, beta, and gamma can be observed in the brain waves depending upon the different functional states of the brain. Any small changes to these waves' frequency patterns help identify neurological conditions $[6,9]$.

\section{EEG Signal Processing}

The dynamically changing functional states of the brain are easily captured in the small variations of the EEG readings. Moreover, the EEG of the normal person also varies from the abnormal person. Therefore, it is very important that these changes be detected by using computer-aided technology to use a variety of signal processing and test mechanisms. This takes place in three steps - preprocessing, feature extraction, and result analysis [6] as shown in Fig. 2. 
Table 2 Brain Rhythms [9].

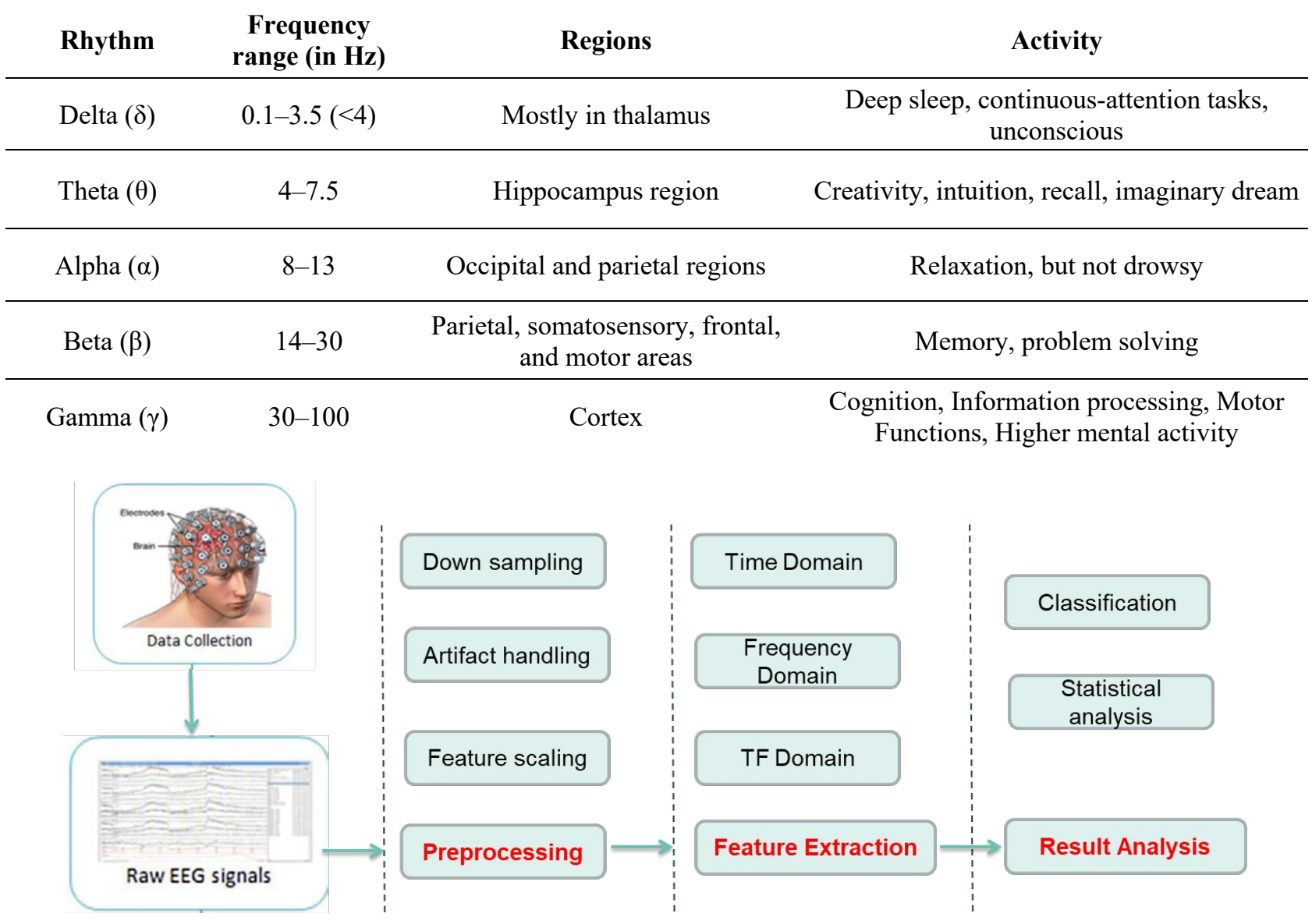

Fig. 2 EEG Signal Processing.

\subsection{Pre-processing}

Due to noise and different artifacts, it is essential to apply pre-processing and denoising methods to decrease their impact on the feature extraction stage. The preprocessing methods applied in the research studies are broadly divided into three categories - down sampling, artifact handling, and feature scaling, as explained below [10].

\subsubsection{Down sampling}

Different EEG devices have different sampling rates. To conserve memory and signal processing time, some of the researchers are down-sampled to certain ranges depending upon the requirement of an application. Down-sampling to $64 \mathrm{~Hz}$ from $512 \mathrm{~Hz}, 256 \mathrm{~Hz}$ to $16 \mathrm{~Hz}$, and $2400 \mathrm{~Hz}$ to $600 \mathrm{~Hz}$, are done in the research studies depending upon the requirement of an application.

\subsubsection{Artifact Handling}

While raw signals of EEG recordings tend to contain noise and artifacts, EEG signals measured from the scalp do not necessarily accurately represent signals originating from the brain. As summarized in Table 3,
Table 3 EEG Artifacts $[9,11]$.

\begin{tabular}{|c|c|c|}
\hline Type & Name & Origin \\
\hline \multirow{3}{*}{$\begin{array}{l}\text { Physiological } \\
\text { Artifacts/Intrinsic }\end{array}$} & EOG & $\begin{array}{l}\text { Eye blink, } \\
\text { eye movement, } \\
\text { eye flatter }\end{array}$ \\
\hline & EMG & $\begin{array}{l}\text { Chewing, } \\
\text { Swallowing, } \\
\text { Clenching, } \\
\text { Talking, sniffing }\end{array}$ \\
\hline & ECG & Heart potentials \\
\hline \multirow{3}{*}{$\begin{array}{l}\text { Non-Physiological } \\
\text { Artifacts/ } \\
\text { Extrinsic }\end{array}$} & Instrumental & $\begin{array}{l}\text { Electrode } \\
\text { misplacement } \\
\text { and cable } \\
\text { movement, } \\
\text { Malfunction of } \\
\text { any part }\end{array}$ \\
\hline & Interference & $\begin{array}{l}\text { High voltage } \\
\text { machine in } \\
\text { surrounding } \\
\text { (Power line) }\end{array}$ \\
\hline & Movements & $\begin{array}{l}\text { Head and body } \\
\text { movement }\end{array}$ \\
\hline
\end{tabular}


artifacts embedded in the EEG recordings are generally subdivided into two categories: physiological and nonphysiological artifacts. Physiological artifacts include electrical potentials elicited by biological activities, which are largely generated from the physical part proximal to the head (e.g., eyes, muscles, and heart) or due to the subjects' movements. Non-physiological Artifacts include the electrode-scalp interface, devices, and environment, which are generated anywhere near the EEG recording system. Variety of artifact removal techniques are used to handle all these kinds of artifacts. A large number of artifacts can be avoided through careful planning of experiments and recording sessions in an appropriate recording environment. Moreover, a variety of methods may also be used to reject noisy data, delete artifacts, and enhance data signal-to-noise ratio (SNR) $[10,12]$. The major problem in the use of these processes is the loss of essential neuronal knowledge from artifactual epochs may lead to incorrect assumptions. Signal based automatic artifact removal algorithms are founded upon some presumption, that each channel is the total sum of pure EEG data and a proportion of an artifact. Several theories have been proposed based on signal transformation, some focusing on various filters for artifact deletion and some are using the blind source separation (BSS) method including a variety of unsupervised learning algorithms without prior information and extra reference channels [11].

The artifact removal techniques have been tabulated in Table 4. The type of artifact removal technique mentioned in the first column, while the rest of the columns denote the type of artifact removed, application in which the corresponding method has been applied with its advantage and limitation.

\subsubsection{Feature Scaling}

Normalization is one of the most widely used methods of function scaling in the literature to demonstrate symmetrical behavior. The scaling is performed either on the raw/filtered values or on the extracted features. For instance, in Ref. [19], the authors use Z-score (mean $=0$, Std Dev =1) before feature extraction similarly in Ref. [8], the authors apply Min-Max and unity method for Multi-class task recognition.

\subsection{Feature Extraction}

Various feature extraction methods are used to perform frequency domain, time domain, and time-frequency domain analysis of the signals $[6,19]$. The feature extraction methods have been categorized into three categories: spectral estimation methods, family of transforms, and time decomposition methods.

Spectral estimation transforms a signal from the time domain to the frequency domain and can provide a description of the signal's power or energy distribution along frequency. Widely used spectral estimation methods of EEG, include Non-parametric (classical): Periodogram, Welch and Parametric and (non-classical): Autoregressive. In spectral analysis, the basic assumption is that the spectrum of an EEG signal is fixed and does not change over time. To reveal the time-varying spectrum of non-stationary EEG signals, time-frequency analysis (TFA) techniques are needed. Some popular methods include the short-time Fourier Transform (STFT), the Continuous Wavelet Transform (CWT) and Discrete Wavelet Transformation (DWT). Similarly, the decomposition method also helps to effectively analyze the signals. The signal is decomposed into a set of intrinsic mode functions (IMFs) that are used as subsignals [8].

\subsection{Result Analysis}

The result analysis phase is explained through classification models and statistical analysis.

Classification Models. A variety of machine learning algorithms are used for the diagnosis of neurological disorders. The machine learning classifiers such as supervised, unsupervised, deep learning neural architectures and ensemble learning models are used for classification purposes in various EEG research studies [8, 20-23]. Some popular classifiers include LDA, SVM with linear and RBF kernel function, KNN, CNN, etc.

Statistical Analysis. Some application studies have used different statistical tests for EEG signal analysis. These tests are applied on features or parameters for different purposes or the application. For instance, T-test, One-Way ANOVA, and Wilcoxon rank-sum test, Kolmogorov-Smirnov test for normal distribution, and non-parametric Kruskal-Wallis test [6].

\section{Diagnosis of Epilepsy}

Epilepsy is a neurological condition that affects the nervous system. It is also known as a seizure disorder. It is usually diagnosed after a person had at least two seizures or after one seizure with a high risk that was not caused by some known medical condition. Epilepsy are divided into two categories [24]: generalized seizure and focal seizure. Subtypes of generalized seizures are absence seizures, tonic seizures; atonic seizures, etc. as is given in Fig. 3.

Epilepsy is caused by a sudden abnormal electrical discharge that occurs in the cerebral networks and usually lasts for less than a few minutes; so, the attacks are hard to predict. Epilepsy occurs in different phases as pre-ictal (immediately preceding seizure), ictal (during a seizure), inter-ictal (in-between seizures) and post-ictal (immediately following a seizure) [22]. EEG records of the epileptic patients are usually long (hours to days) and contain an immense amount of patient info. A visual assessment of such recordings is time-consuming, slow, and sensitive to errors and subjects to inter-observer variability [25]. Hence, several methods have been developed to help diagnose epileptic seizures by automatic computer-assisted therapy (CAD). EEG is commonly used for the diagnosis and analysis of epilepsy [8]. EEG helps to assess the types of seizure and epileptic condition of patients with epilepsy. The EEG finding makes a significant contribution to determine a 
Table 4 Artifact removal techniques.

\begin{tabular}{|c|c|c|c|c|c|}
\hline Sr. No & Method & Description & $\begin{array}{l}\text { Type of } \\
\text { artifact }\end{array}$ & Advantage & Limitation \\
\hline 1 & $\begin{array}{l}\text { Regression } \\
\text { Methods } \\
{[10-12]}\end{array}$ & $\begin{array}{l}\text { It is applied under the } \\
\text { assumption: each channel } \\
\text { is the cumulative sum of } \\
\text { pure EEG data and a } \\
\text { proportion of artifact }\end{array}$ & $\begin{array}{l}\mathrm{EOG} \\
\mathrm{ECG}\end{array}$ & $\begin{array}{l}\text { - computationally } \\
\text { simple }\end{array}$ & $\begin{array}{l}\text { - requires exogenous } \\
\text { reference channels, } \\
\text { outcomes are highly } \\
\text { affected by } \\
\text { bidirectional } \\
\text { contamination; } \\
\text { - it does not work } \\
\text { successfully on other } \\
\text { artifacts; } \\
\text { - cannot perform on } \\
\text { single channel }\end{array}$ \\
\hline
\end{tabular}

\begin{tabular}{|c|c|c|c|c|c|}
\hline 2 & $\begin{array}{l}\text { Wavelet } \\
\text { Transform } \\
{[10-13]}\end{array}$ & $\begin{array}{l}\text { Threshold applied to } \\
\text { discard the signal that } \\
\text { contain artifacts }\end{array}$ & $\begin{array}{l}\text { EOG } \\
\text { EMG }\end{array}$ & $\begin{array}{l}\text { - very good time } \\
\text { frequency localization } \\
\text { features relative to } \\
\text { Fourier transform }\end{array}$ & $\begin{array}{l}\text { - cannot remove } \\
\text { artifacts completely if } \\
\text { the spectral properties } \\
\text { overlap with the } \\
\text { spectral properties of } \\
\text { the artifacts }\end{array}$ \\
\hline
\end{tabular}

\begin{tabular}{|c|c|c|c|c|}
\hline & & \multicolumn{3}{|c|}{ BSS } \\
\hline 3 & $\begin{array}{l}\text { Principal } \\
\text { Component } \\
\text { Analysis } \\
\text { (PCA) } \\
{[10-14]}\end{array}$ & $\begin{array}{l}\text { Based on Eigenvalues of } \\
\text { covariance matrix }\end{array}$ & EOG & $\begin{array}{l}\text { - is more } \\
\text { computationally } \\
\text { efficient than linear } \\
\text { regression methods }\end{array}$ \\
\hline
\end{tabular}

\begin{tabular}{|c|c|c|c|c|}
\hline 4 & $\begin{array}{l}\text { Independent } \\
\text { Component } \\
\text { Analysis } \\
\text { (ICA) } \\
{[10-14]}\end{array}$ & $\begin{array}{l}\text { Assuming that signal } \\
\text { sources are instantaneously } \\
\text { linear mixtures of cerebral } \\
\text { and artifactual sources can } \\
\text { decompose observed signal } \\
\text { into independent } \\
\text { components (ICs). Once } \\
\text { ICs are extracted from } \\
\text { original signals, the clean } \\
\text { signal reconstructed by } \\
\text { discarding ICs contains } \\
\text { artifacts }\end{array}$ & $\begin{array}{l}\text { EMG } \\
\text { ECG } \\
\text { EOG }\end{array}$ & $\begin{array}{l}\text { - does not require any } \\
\text { prior information or } \\
\text { additional reference } \\
\text { channel for removal of } \\
\text { artifacts; } \\
\text { - shown promising } \\
\text { results in cases where } \\
\text { the neuronal and } \\
\text { artifactual sources are } \\
\text { not completely } \\
\text { independent }\end{array}$ \\
\hline
\end{tabular}

$\begin{array}{lll}\text { 5 Canonical } & \text { Use second order statistics, EMG } \\ \text { Correlation } & \text { which bring shorter } \\ \text { Analysis } & \text { computational time. It } \\ & \text { finds the linear relation } \\ & \text { between two multi- } \\ & \text { dimensional random } \\ & \text { variables by maximizing } \\ & \text { the pairwise correlations } \\ & \text { across the two data sets }\end{array}$

- uses second-order statistics with less computational cost than ICA;

- does not require the assumptions of orthogonality and Gaussian distributions
- fails to separate the interferences when the potential of drifts and EEG data are similar; fails on similar amplitude for neuronal and artifactual activity require the assumptions of orthogonality and Gaussian distributions - cannot perform on single channel; - the complex iterative procedure limits use in online/real time applications; - disregard of temporal or spatial relations within sources will result in the loss of relevant information;

- require the assumptions of orthogonality and Gaussian distributions - cannot perform on single channel 
6

Morphological component analysis

(MCA)

$[15,16]$
Used to decompose a signal into components that have different morphological aspects, signals can be represented as a linear combination of three morphological components using MCA full form theory

7 Empirical Heuristic technique. for Mode non-stationary and nonDecomposition linear signal processing It $[11,17]$ decomposes/extracting the signal, $x[n]$, into a set of components with amplitude and frequency modulated, $b[n]$, patterns from time series data called intrinsic mode functions (IMFs)
EOG

- fast computation of the estimation of the signal coefficients using the basis pursuit algorithm, less memory requirement
- always requires a database containing morphologies of different types of artifacts, and therefore, its performance is highly dependent on the available templates of artifacts

$\begin{array}{lll}\text { EMG } & \bullet \text { this technique is } & \bullet \text { it is very sensitive } \\ & \text { empirical and data } & \text { for noise which incurs } \\ \text { driven technique, } & \text { mode mixing }\end{array}$
whereas other methods depend on the selections of basic functions, such as wavelet analysis

\section{Filtering Methods}

\begin{tabular}{|c|c|c|c|c|}
\hline 8 & $\begin{array}{l}\text { Adaptive } \\
\text { Filtering } \\
{[11,12]}\end{array}$ & $\begin{array}{l}\text { To quantize the amount of } \\
\text { artifactual contamination } \\
\text { in the primary input, by } \\
\text { iteratively adjusting the } \\
\text { weights according the } \\
\text { optimization algorithm, } \\
\text { and subtract it from EEG } \\
\text { with artifacts signals }\end{array}$ & $\begin{array}{l}\mathrm{EOG} \\
\mathrm{ECG}\end{array}$ & - \\
\hline \multirow[t]{3}{*}{9} & $\begin{array}{l}\text { Wiener } \\
{[11,18]}\end{array}$ & $\begin{array}{l}\text { It is a linear statistical } \\
\text { filtering technique used to } \\
\text { estimating the true EEG } \\
\text { data with the purpose to } \\
\text { develop a linear time } \\
\text { invariant filter to minimize } \\
\text { the mean square error } \\
\text { between the pure EEG data } \\
\text { and the estimated signal }\end{array}$ & $\begin{array}{l}\text { EOG } \\
\text { EMG }\end{array}$ & $\begin{array}{l}\text { - minimization is } \\
\text { done using an } \\
\text { estimation of the } \\
\text { power; } \\
\text { - spectral densities of } \\
\text { the signal and artifact; } \\
\text { hence it does not need } \\
\text { a reference waveform }\end{array}$ \\
\hline & & $\begin{array}{l}\text { Focal Seizure } \\
\text { •Focal aware seizures } \\
\text { •Focal impaired awaren }\end{array}$ & s seizures & \\
\hline & & $\begin{array}{l}\text { Generalized Seizur } \\
\text {-Absence Seizures } \\
\text {-Tonic Seizures } \\
\text {-Atonic Seizures } \\
\text {-Myoclonic Seizures } \\
\text { - Clonic Seizures } \\
\text {-Tonic-Clonic Seizures }\end{array}$ & & \\
\hline
\end{tabular}

Fig. 3 Types of epilepsy.

- reference channel is given, as one of the inputs to the filter; additional sensors are needed to provide reference inputs

- calibration needed prior to usage and that it cannot run in real time 
multi-axial diagnosis of epilepsy, in terms of whether the seizure disorder is idiopathic or symptomatic, focal or generalized, part of a specific epilepsy syndrome [8].

\subsection{Classification and prediction of seizure}

EEG based epileptic seizure detection methods stem from the observation that EEG signal descriptors allow discrimination between normal and abnormal brain activity. A variety of EEG signal processing methods are available for the accurate detection of brain seizures. In EEG, signal analysis for epilepsy, classification / detection is done between epileptic and healthy subjects. Various approaches like supervised, unsupervised approaches are used to discriminate categories as normal vs. ictal, normal vs. pre-ictal, preictal vs. ictal, focal vs. non-focal, seizure vs. nonepileptic etc.

Most methods employ classification techniques from the supervised machine. Among these methods, Support Vector Machine (SVM) is used more extensively as a supervised machine learning based classifier. In study [23], the authors employed a LS-SVM methodology, which proposes the use of decomposed EEG signals into six sub-bands namely D1-D5 and A5 using DWT. Then, the author uses approximate entropy (ApEn) feature, which performs the study of variation in subbands as an input function for SVM. Many researchers have used different types of SVM such as SVM (RBF), LS-SVM [26] with different feature extraction methods like PCA, wavelet transform (WT), ICA. Likewise, prediction of epileptic seizure is done by analyzing time series EEG signals using k-nearest neighbors (KNN) classifier [27].

In the same way random forest, back proposition, generalized relevance learning vector quantization (GRIVQ), WT with random forest, WT with SVM, fuzzy logic and, wavelet-bank filtering and dimensionality reduction using a generalized Gaussian distribution (GGD) with LDA $[23,28]$ are also used to classify epileptic signals. Focal epilepsy is a variant of epilepsy that mostly occurs in the limited area of the brain. Focal EEG signals are recorded from the brain area where first ictal EEG signal changes are detected and non-focal signals are recorded wherein, they do not take part in the seizure onset. In study [29], the authors apply empirical wavelet transform (EWT) with LS-SVM to separate the focal and non-focal rhythms from groups of EEG signals.

Neurologists are able to diagnose whether a person has epilepsy by monitoring EEG manually. However, it is also important to discriminate between the inter-ictal and ictal stages so that proper actions can be taken timely to avoid the risks of epilepsy. In Ref. [30], the authors discriminate between the inter-ictal and ictal EEGs using multi-basis maximal overlap discrete wavelet package transform MODWPT, six dimensionality reduction algorithms, and LS-SVM.

In the conventional methods where data is represented by features, there is a great loss of data.
Feature extraction becomes very complex due to the presence of noise and artifacts in data. Thus, it is a challenging problem to produce a generalized automatic system with loyal performance, especially with low data availability. Currently, deep learning approaches substitute methods that integrate few sections of the data in the classification. The EEG signal can be processed as raw [31]. In Ref. [32], the authors apply a Deep Convolutional Neural Network (DCNN) on the raw EEG signals for the automatic identification of differentiated spatial features between inter-ictal and pre-ictal states and then used Bi-LSTM features that are created by the DCNN to classify inter-ictal or preictal segments of the incoming EEG section.

Early prediction is done by analyzing pre-ictal and intertidal stages. Various ML and DL are used for early prediction $[22,33]$. These analyses are evaluated based on prediction time or other metrics like seizure occurrence period (SOP), the time duration in which there is a possibility of seizure and seizure prediction horizon (SPH), the duration of time between the alarm and the start of SOP.

\subsection{Real time monitoring system}

In real-time monitoring EEG recording and video surveillance videos are collected from epilepsy patients with some prior knowledge (label the different states of epilepsy patients from doctors). An expert observes the EEG recording and labels the seizure with the assistance of monitoring videos. It is based on change detection or event detection approaches, which find the change between brain states wherein the EEG changes from normal state to abnormal state [34].

Similarly, various warning systems are also available. Such systems utilize both ECG and EEG data to generate an alarm before seizure onset according to an ideal situation. Some systems combine accelerometer and electrodermal activity methods with several electronic mobile applications developed to electronically track seizure information Seizure record applications allow families to easily record seizures in an electronic format that is user friendly, mobile, and easily accessed by their treating epileptologist [35].

\subsection{Comparative result Analysis for Epilepsy classification and Prediction}

The various seizure classification methodologies are discussed in the earlier section. It is imperative to analyze the statistical evaluation of these methods' performance. The summary of methods, features used, the dataset used, and their performance in terms of accuracy is given in Table 5 According to the state-of-the-art, it has been observed that various methods perform differently for the different datasets. For instance, LS-SVM gives 100\% accuracy for Bonn dataset [36] but it gives $90 \%$ for the Bern-Barcelona database [37]. Deep learning approaches are more robust irrespective of the dataset. 
Table 5 Comparison of studies on classification for epilepsy.

\begin{tabular}{|c|c|c|c|c|c|}
\hline Sr. no & $\begin{array}{c}\text { Year of } \\
\text { Publication }\end{array}$ & Dataset & Features & Method & $\begin{array}{c}\text { Overall } \\
\text { Accuracy } \\
(\%)\end{array}$ \\
\hline \multicolumn{6}{|c|}{ Bonn dataset [36] } \\
\hline 1 & $\begin{array}{l}{[23]} \\
(2017)\end{array}$ & & DWT based ApEn & $\begin{array}{l}\text { LS-SVM, SVM (RBF) for } \\
\text { (A-E, AB-E, CE, CD-E, } \\
\text { ABCD-E) }\end{array}$ & 100 \\
\hline 2 & $\begin{array}{c}{[25]} \\
(2019)\end{array}$ & & $\begin{array}{l}\text { Discrete Wavelet + } \\
\text { Arithmetic coding }\end{array}$ & $\begin{array}{l}\text { k-NN, Naïve Bayes, MLP, } \\
\text { and SVM for (A-E, AB-E, } \\
\text { C-E, CD-E, ABCD-E) }\end{array}$ & 100 \\
\hline \multirow{5}{*}{3} & \multirow{5}{*}{$\begin{array}{c}{[26]} \\
(2018)\end{array}$} & \multirow{5}{*}{$\begin{array}{c}\text { Five sets marked } \\
\text { as (AE) }\end{array}$} & \multirow{5}{*}{$\begin{array}{l}\text { Principal Component } \\
\text { Analysis (PCA) and } \\
\text { Wavelet Transform }\end{array}$} & Backpropagation & 95.29 \\
\hline & & & & GRLVQ & 98.66 \\
\hline & & & & WT+Randome Forest & 98.05 \\
\hline & & & & PCA+Randome Forest & 98.22 \\
\hline & & & & PCA+GRLVQ & 98.45 \\
\hline \multirow{4}{*}{4} & \multirow{4}{*}{$\begin{array}{c}{[30]} \\
(2019)\end{array}$} & \multirow{4}{*}{$\begin{array}{c}\text { Subject: } \\
\text { 5-HC (A, B), } \\
\text { 5-preictal (C, D), } \\
\text { 5-Ictal (E) } \\
\text { Channel: 1 } \\
\text { Sampling rate: } \\
\text { 173.61 Hz }\end{array}$} & \multirow{4}{*}{$\begin{array}{l}\text { Maximal overlap } \\
\text { discrete wavelet } \\
\text { package transform } \\
\text { (MODWPT) }\end{array}$} & LS-SVM & \\
\hline & & & & $\begin{array}{l}\text { Multi-basis } \\
\text { MODWPT+PCA+ } \\
\text { LK-LSSVM/ } \\
\text { RK-LS-SVM }\end{array}$ & $99.60 / 99.20$ \\
\hline & & & & $\begin{array}{l}\text { Multi-basis } \\
\text { MODWPT+ICA+ } \\
\text { LK-LSSVM/ } \\
\text { RK-LS-SVM }\end{array}$ & $99.76 / 98.80$ \\
\hline & & & & $\begin{array}{l}\text { Multi-basis } \\
\text { MODWPT+LLE+ } \\
\text { LK-LSSVM/ } \\
\text { RK-LS-SVM }\end{array}$ & $98.73 / 98.13$ \\
\hline 5 & $\begin{array}{c}{[31]} \\
(2019)\end{array}$ & & NA & $\begin{array}{l}\text { CNN + Scalogram for } \\
\text { A-E, } \\
\text { A-D, } \\
\text { D-E, } \\
\text { AD-E, } \\
\text { A-B-C-D }\end{array}$ & $\begin{array}{c}99.5 \\
100 \\
98.5 \\
99 \\
93.6\end{array}$ \\
\hline
\end{tabular}

Boston database [38]

\begin{tabular}{|c|c|c|c|c|c|}
\hline 6 & $\begin{array}{c}{[28]} \\
(2018)\end{array}$ & $\begin{array}{l}\text { Subject: } \\
\text { considered } 39 \\
\text { signals related to } \\
13 \text { seizures and } \\
26 \text { non-seizure } \\
\text { signals } \\
\text { Channel: } 23 \\
\text { Sampling rate: } \\
2561 \mathrm{~Hz}\end{array}$ & $\begin{array}{l}\text { Separation of brain } \\
\text { rhythms: wavelet } \\
\text { bank filtering, } \\
\text { statistical model } \\
\text { based on } \\
\text { dimensionality } \\
\text { reduction with GGD }\end{array}$ & $\begin{array}{l}\text { Linear discriminant analysis } \\
\text { for } \\
\text { Delta band, } \\
\text { Theta band, } \\
\text { Alpha band, } \\
\text { Beta band, } \\
\text { Gamma band }\end{array}$ & $\begin{array}{c}95.13 \\
92.69 \\
96.59 \\
94.48 \\
97\end{array}$ \\
\hline 7 & $\begin{array}{c}{[32]} \\
(2018)\end{array}$ & $\begin{array}{l}\text { Subject: } \\
13 \text { seizures and } \\
26 \text { non-seizures } \\
\text { Channel: } 23 \\
\text { Sampling rate: } \\
2561 \mathrm{~Hz}\end{array}$ & NA & $\mathrm{DCNN}+\mathrm{BiLSTM}$ & 99.66 \\
\hline
\end{tabular}




\section{Bern-Barcelona database [37]}

\begin{tabular}{|c|c|c|c|c|}
\hline 8 & $\begin{array}{c}{[29]} \\
(2016)\end{array}$ & $\begin{array}{l}\text { Subject: } \\
5 \text { intracranial } \\
\text { Channel: } 64 \\
\text { Sampling rate: } \\
512 \mathrm{~Hz}\end{array}$ & $\begin{array}{l}\text { Area computed from } \\
\text { 2D Reconstructed } \\
\text { phase space plot } \\
\text { (RPS) of rhythms } \\
\text { using CTM }\end{array}$ & $\begin{array}{l}\text { Least-squares support vector } \\
\text { machine (LSSVM) for } \\
50 \text { pairs of signals area, } \\
750 \text { pairs of signals }\end{array}$ \\
\hline
\end{tabular}

attention, listening attentively, following commands,

\subsection{Datasets for Epilepsy Analysis and Prediction}

EEG is a significant means for the identification, analysis, and prediction of epilepsy seizures. For these reasons, multiple open-access databases have been published by different hospitals and research centers. For instance, the Center of Epilepsy at Children's Hospital, Boston, and Temple University Hospital have made their EEG databases publicly available to the researchers. The database of Bonn University is not large enough but is extensively used for the detection of ES in the literature. It consists of 5 datasets A, B, C, D, and E. CHB-MIT database has data of 22 patients with 9-24 recordings of each patient and every recording is $1 \mathrm{~h}$ long with some discontinuities due to hardware limitation (some cases have $2-4$ h long recordings) [23, 25]. Freiburg Hospital's database was one of the most notable databases, which contained iEEG data of 21 subjects with around 88 seizures, but recently it has been merged into EPILEPSIAE database to provide larger datasets due to which this database is not open-source now [22].

Bern-Barcelona Database containing intracranial EEG signals. The EEG signals were recorded from 5 epilepsy patients who were the candidates for surgery due to long-standing drug-resistant temporal lobe epilepsy. The database contains 3750 pairs of EEG signals for each focal and non-focal category. Each pair contains EEG signals, namely $x$ and $y$, which were recorded from adjacent channels. The EEG signals were sampled at a rate of $512 \mathrm{~Hz}$, and the duration of each signal is $20 \mathrm{sec}$ [29]. Likewise, CHB-MIT Scalp EEG Database collected at the Children's Hospital Boston. Recordings, grouped into 23 cases, were collected from 22 subjects. Subjects were monitored for up to several days following withdrawal of anti-seizure medication in order to characterize their seizures and assess their candidacy for surgical intervention. All signals were sampled at 256 samples per second with 16-bit resolution [30].

\section{Diagnosis of ADHD}

ADHD is a common neuro-developmental disorder that begins in childhood and can continue through adolescence and adulthood. ADHD in children leads to a lack of interest in all things. The dilemma of the syndrome concerns children in their families, in the study area, and in the community. Kids have problems paying keeping still. ADHD is divided into three types: Inattentive, Hyperactive/Impulsive, and combined (Fig. 4). Symptoms differ according to type [39, 40].

Diagnosis of ADHD is typically carried out using clinical interviews structured around the diagnosis classification systems of DSM-5 and ICD-11 [40-42]. Different diagnosis and analysis methods for ADHD are found in the literature. A more comprehensive description can be found in study [43]. Some specific symptoms of the disorder are simpler to identify than other disorder's symptoms. It is needed to conduct a diagnosis procedure followed by evaluation techniques for the disorder. Among all diagnosis methodology, EEG plays a key role to assess and evaluate the ADHD disorder. Human activities are related to neural functions. Many researchers adopted this main tool to diagnose ADHD in their research.

Numerous EEG studies utilized spectral analyses to reveal the EEG characteristics features to describe the difference between attentive and non-attentive states of ADHD children. Likewise, assessment of cognitive capability is also focused in ADHD-affected children.

\subsection{Classification of EEG signals for ADHD}

Machine learning methods can be used for finding some relevant discriminators between ADHD and control groups with different EEG characteristics viz. spectral features, nonlinear features, statistical features.

The majority of EEG analysis for ADHD is based on spectral power features which are measured with the Theta/beta ratio (TBR), Theta/alpha ratio, TBAR (Theta/ (alpha + beta)), relative delta, theta, alpha, and beta power $[4,41]$.

In literature, many researchers have utilized different methods to analyze resting-state EEG (rsEEG) and time series recorded from an ADHD group and a control group. Authors $[44,45]$ contributed to discovering the best indicator of attention for ADHD. They applied the wavelet decomposition method to obtain different frequency bands of EEG. They extracted seven features (relative Delta, Theta, Alpha and Beta power, TBR, TAR, and TBAR) for each sample. Then, linear discriminant analysis (LDA) was used for classification. They proved that the TBR is a significant feature in discriminating between attentive and non-attentive states. Likewise [41], used relative spectral power, spectral power ratio, complexity analyses, and bicoherence to extract rsEEG features. To examine the 
effect of these features on ADHD identification, they achieved an AUC of 0.9158 and an accuracy of $84.59 \%$ using a support vector machine (SVM).

TBR has been used as a measure for discriminating between children with and without ADHD in several studies. However, in some cases, researchers could not attain good accuracy using TBR. In study [39], the authors attempted 0.878 AUC using eight key feature descriptors for the ADHD/control classification and ranked based on the t-test. The results for beta (indicative of heightened cortical arousal) activity has been less consistent, with some studies reporting reduced beta activity in frontal and central regions and others not. There also tends to be a small number of ADHD children who display an abundance of beta activity [46]. In Refs. [20,21], the authors apply machine-learning techniques for diagnosis of adult ADHD.

With the spectral features of EEG, non-linear features also play a vital role in ADHD analysis. Non-linear analysis of EEG waves has revealed new information on the complex dynamics of underlying neural

Table 6 Comparison of studies on classification for ADHD. networks [47]. Well-known entropy methods like Sample Entropy (SampEn), Dispersion entropy (DispEn), Multivariate Sample Entropy (mvSE), Approximate entropy, sample entropy are used to classify EEG signals [48]. Likewise, in study [49], fractal dimension as Higuchi's, Katz's, and Petrosian's has been applied to discriminate ADHD and healthy controls. Brief comparison of studies on classification for ADHD is given in Table 6 .

\subsection{Assessment of Cognitive Capability}

EEG can be used for the assessment of the attention capability [51] and cognitive capability of ADHDaffected children. Based on this, new methodology or techniques of teaching can be adapted to enhance their learning capability. In order to investigate human cognitive patterns, Event-related potentials (ERPs) visual stimulation with the Oddball paradigm is used as the basis.

\begin{tabular}{ccl} 
Sr. no & $\begin{array}{c}\text { Year of } \\
\text { Publication }\end{array}$ & \multicolumn{1}{c}{ Dataset } \\
\hline & & \\
1 & {$[39]$} & $\begin{array}{l}\text { Subject: } 30 \mathrm{ADHD}, 30 \mathrm{HC} \\
\text { Channel: } 21 \\
\text { Sampling rate: } 256 \mathrm{~Hz} \\
\text { Recording: Resting state }\end{array}$ \\
& 2019 &
\end{tabular}

\begin{tabular}{|c|c|c|}
\hline 2 & $\begin{array}{c}{[45]} \\
(2017)\end{array}$ & $\begin{array}{l}\text { Subject: } 120 \\
\text { Channel: } 1 \\
\text { Sampling rate: } 256 \mathrm{~Hz} \\
\text { Recording: Working state }\end{array}$ \\
\hline 3 & $\begin{array}{c}{[47]} \\
(2016)\end{array}$ & $\begin{array}{l}\text { Subject: } 30 \text { ADHD, } 30 \mathrm{HC} \\
\text { Channel: } 19 \\
\text { Sampling rate: } 256 \mathrm{~Hz} \\
\text { Recording: Working state }\end{array}$ \\
\hline
\end{tabular}

\section{Eight crucial feature descriptors were selected and ranked based on the t-test (refer reference for more detail)}

Overall

best

Accuracy

(\%)

Feature Selection using
Mutual Information

Fractal dimension (FD)

based features:

Higuchi, Katz and

Petrosian fractal Multi-Layer

dimensions, largest Perceptron

(MLP)

93.65
Lyapunov exponent and approximate entropy

\begin{tabular}{|c|c|c|c|c|c|}
\hline \multirow[b]{2}{*}{4} & \multirow{2}{*}{$\begin{array}{c}{[49]} \\
(2020)\end{array}$} & \multirow{2}{*}{$\begin{array}{l}\text { Subject: } 23 \text { ADHD, } 23 \mathrm{HC} \\
\text { Channel: } 4 \\
\text { Sampling rate: } 2500 \mathrm{HZ} \\
\text { Recording: Working state }\end{array}$} & Morphologic Features & $\begin{array}{c}\text { SVM } \\
\text { k-NN } \\
\text { RF }\end{array}$ & $\begin{array}{c}89.13 \\
86.95 \\
82.6\end{array}$ \\
\hline & & & $\begin{array}{l}\text { Non-linear Features, } \\
\text { Wavelet }\end{array}$ & $\begin{array}{l}\text { AdaBoost } \\
\text { MLP } \\
\text { NB } \\
\text { LR }\end{array}$ & $\begin{array}{l}89.13 \\
91.3 \\
86.95 \\
89.13\end{array}$ \\
\hline 5 & $\begin{array}{c}{[50]} \\
(2019)\end{array}$ & $\begin{array}{l}\text { Subject: } 50 \text { ADHD, } 51 \mathrm{HC} \\
\text { Channel: } 128,32 \text { selected } \\
\text { Sampling rate: } 1000 \mathrm{HZ} \\
\text { Recording: Resting state }\end{array}$ & $\begin{array}{l}\text { DF, Brain Network } \\
\text { Measures }\end{array}$ & $\begin{array}{l}\text { CNN } \\
\text { MLP } \\
\text { SVM }\end{array}$ & $\begin{array}{l}94.67 \\
84.53 \\
84.17\end{array}$ \\
\hline
\end{tabular}


For instance, the probable region of the brain that shows abnormality due to ADHD syndrome can be used for assessment of learning capability of ADHD-affected children [43]. Similarity patients are asked to do some motor activity like to arrange the spoons and hand them over to their caretaker. Moreover, visual cues activity as colors, numbers, and animals, are identified to find out the attentive and cognitive capability [44].

Cognitive training is a commonly used therapy for ADHD. However, the main challenges with such training are illuminating the repetitive scenes, stimulating the user's interests, encouraging more engagement, and adjusting the difficulty level. Applying EEG signal is a familiar method towards the improvements in the sustained attention levels of children by designing video games [52] with the introduction of "serious games" attempted to measure attention to detect ADHD.

\subsection{Datasets for ADHD Analysis}

An extensive amount of literature has been researched by collaborating with the various medical associations. Data used for such research are not publicly available due to privacy issues. However, some research has been done on ADHD analysis on publicly available data.

EEG data for ADHD/Control children done EEG recording for 61 children with ADHD and 60 healthy controls (boys and girls, ages 7-12) is given in Ref. [47]. The ADHD children were diagnosed by an experienced psychiatrist with DSM-IV criteria, and have taken Ritalin for up to 6 months. EEG recording was performed based on 10-20 standard by 19 channels (Fz, Cz, Pz, C3, T3, C4, T4, Fp1, Fp2, F3, F4, F7, F8, P3, P4, T5, T6, O1, O2) at $128 \mathrm{~Hz}$ sampling frequency. The $\mathrm{A} 1$ and $\mathrm{A} 2$ electrodes were the references located on earlobes. Two electrodes that were placed below and above the right eye recorded the eye movement. EEG recording protocol was based on visual attention tasks. In the task, a set of pictures of cartoon characters was shown to the children and they were asked to count the characters.

Similarly, in FOCUS [52], EEG brain recording of ADHD and non-ADHD individuals during gameplay of a brain-controlled game, recorded with an EMOTIV EEG headset are collected. It can be used to design and test methods to detect individuals with ADHD.

\section{Diagnosis of Alzheimer's disease (AD)}

Dementia is a syndrome usually of a chronic or progressive nature in which there is deterioration in cognitive function. Worldwide, around 50 million people have dementia [53]. Alzheimer's disease is the most common form of dementia.

Alzheimer's disease is an irreversible, progressive brain disorder that slowly destroys memory and thinking skills, and, eventually, the ability to carry out the simplest tasks. In most people with Alzheimer's, symptoms (Fig. 5) first appear in their mid-60s. Estimates vary, but experts suggest that more than 5.5 million Americans, most of them age 65 or older, may have dementia caused by Alzheimer's.

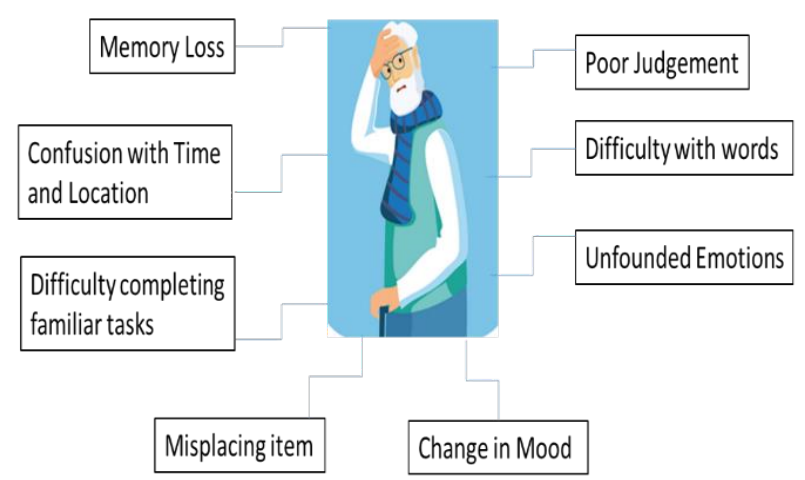

Fig. 5 Alzheimer's Symptoms.

Numerous neuroimaging techniques have been used with the aim of distinguishing $\mathrm{AD}$ and Mild Cognitive Impairment (MCI) patients from cognitively healthy control (HC) subjects: positron emission tomography (PET), magnetic resonance spectroscopy, functional magnetic resonance imaging (fMRI), magnetoencephalography (MEG), and electroencephalography (EEG) [54]. PET and fMRI show a good structural accuracy, but both offer a limited temporal resolution. By contrast, EEG and MEG are noninvasive techniques with high temporal resolution, allowing for studying the dynamic processes involved in the regulation of complex functional brain systems [55]. Particularly, EEG is widely used due to its portability, low cost, and availability. Moreover, EEG has already shown its usefulness to characterize brain dynamics in $\mathrm{AD}$ and $\mathrm{MCI}$.

\subsection{Early Diagnosis and classification}

To diagnose Alzheimer using EEG, methods are divided into two approaches. The first accomplished in the resting state (awake at rest) in the absence of any stimulus. Since the patient is not required to perform a behavioral task, it is more comfortable and less stressful for patients. The second approach to EEG studies is conducted when the subject is performing a pre-defined task (task-oriented). Approach of task-oriented EEG studies is not ideal for most people with $\mathrm{AD}$ since patients have an increase of anxiety and anger.

The discrimination of early Alzheimer's disease (AD) and its prodromal form (i.e., mild Cognitive impairment, $\mathrm{MCI})$ from cognitively healthy control (HC) subjects is crucial since the treatment is more effective in the first stages of the dementia. MCI is an early stage of Alzheimer's or another dementia. For early diagnosis in Refs. [54,56], compared behavioral results (reaction time and accuracy), ERP and ERD/ERS responses when healthy elderly (HE) controls, MCI and mild AD patients were performing a three-level N-Back visual working memory task.

The anomalies that $\mathrm{AD}$ and $\mathrm{MCI}$ induce in EEG operation have been usually studied using simple signal processing approaches, such as spectral techniques via a power increase in low frequency bands, as well as a decrease in higher frequencies. Nonlinear analysis [57] 
as permutation entropy methods have also been widely used in order to provide complementary details to spectral measures.

In Ref. [38], a combination of spectral measures and nonlinear methods applied to evaluate the diagnostic usefulness of an EEG-based methodology by means of different multiclass classifiers: quadratic discriminant analysis QDA, multi-layer perceptron neural network (MLP), and logistic discriminant analysis (LDA) is undertaken. The Relative Power (RP) in Deep Neural Network metric enumerates the abnormal EEG pattern and distinguishes HC and MCI. In Ref. [58], the authors analyze the Electroencephalogram (EEG) signal, extract the features using Fast Fourier Transform (FFT) and classify the disease by CNN with good accuracy. A brief comparative study for result analysis for Alzheimer's classification is shown in Table 7.

\subsection{Dataset for Alzheimer's disease}

The most widely used databases like ADNI (http://adni.loni.usc.edu), AIBL (http://aibl.csiro.au), and OA-SIS (www.oasis-brains.org) [54] provides clinical, genetic, MRI images open source databases for Alzheimer's. Most researchers have used EEG data recording with the permission of a clinical institution; in this case, data can be available upon request. As in Ref. [62], the database was recorded in real clinical conditions at Charles-Foix Hospital (Ivry-sur-Seine,
France), data is available by mail request. Likewise, some researchers publish their data for research use, as in the study was conducted on mild $\mathrm{AD}$ and normal participants. This data includes EEG from 4 channels (Fp1-FzCz-Pz) with A1 earlobe as reference. The sampling frequency is $200 \mathrm{~Hz}$.

\section{Discussion}

We conducted a comprehensive study for diagnosis of Epilepsy, ADHD and Alzheimer. This study demonstrated that EEG characteristics could be used for diagnosis of neurological disorders, but we found several limitations and issues also. The selection of methodologies used to compare the research between them is a significant challenge when examining the literature. Differences in the selection of participants, EEG acquisition method and interpretation, could influence performance. This lack of standards presents a general confound for the field that extends beyond the implications for particular study.

The core problem is the limited number of participants with respect to the large number of features and tests that have been applied, therefore overfitting may be unavoidable $[25,62]$. Likewise, heterogeneity in etiology, symptoms and treatment outcomes have affected the accuracy of EEG analysis. Symptoms also change over time as the subject ages; features of EEG change accordingly.

Table 7 Comparison of Studies on classification for Alzheimer's.

\begin{tabular}{|c|c|c|c|c|c|}
\hline Sr.no & $\begin{array}{c}\text { Year of } \\
\text { Publication }\end{array}$ & Dataset & Features & Methods & $\begin{array}{c}\text { Overall best } \\
\text { Accuracy } \\
\text { (\%) }\end{array}$ \\
\hline \multirow{6}{*}{1} & \multirow{6}{*}{$\begin{array}{c}{[55]} \\
(2018)\end{array}$} & \multirow{6}{*}{$\begin{array}{l}\text { Subject: } 37 \mathrm{AD}, 37 \mathrm{MCI} \text {, } \\
\text { and } 37 \text { elderly } \mathrm{HC} \\
\text { Channel: } 19 \\
\text { Sampling rate: } 200 \mathrm{~Hz} \\
\text { Recording: Resting state }\end{array}$} & \multirow{6}{*}{$\begin{array}{c}\text { Spectral and } \\
\text { nonlinear }\end{array}$} & LDA & 76.47 \\
\hline & & & & QDA & 78.43 \\
\hline & & & & MLP for HC vs. All & 78.43 \\
\hline & & & & LDA & 74.51 \\
\hline & & & & QDA & 74.51 \\
\hline & & & & MLP for AD vs. Al & 76.47 \\
\hline
\end{tabular}

\begin{tabular}{|c|c|c|c|c|c|}
\hline 2 & $\begin{array}{c}{[59]} \\
(2016)\end{array}$ & $\begin{array}{l}\text { Subject: } 50 \mathrm{AD}, 50 \mathrm{HC} \\
\text { Channel: } 19 \\
\text { Sampling rate: } 1024 \mathrm{~Hz} \\
\text { Recording: Resting state }\end{array}$ & $\begin{array}{c}\text { ICA/Wavelet/ } \\
\text { Spectral }\end{array}$ & SVM & 96 \\
\hline \multirow{8}{*}{3} & \multirow{8}{*}{$\begin{array}{c}{[60]} \\
(2019)\end{array}$} & \multirow{8}{*}{$\begin{array}{l}\text { Subject: } 63 \mathrm{AD}, 63 \mathrm{MCI} \text {, } \\
63 \mathrm{HC} \\
\text { Channel: } 19 \\
\text { Sampling rate: } 1024 \mathrm{~Hz} \\
\text { Recording: Resting state }\end{array}$} & \multirow{4}{*}{ Epoch } & $\mathrm{CNN}$ for $\mathrm{AD}$ vs. $\mathrm{HC}$ & 92.95 \\
\hline & & & & AD vs. $\mathrm{MCI}$ & 84.62 \\
\hline & & & & MCI vs. HC & 91.99 \\
\hline & & & & AD vs. MCI vs. HC & 83.3 \\
\hline & & & \multirow{4}{*}{ PSD } & $\mathrm{CNN}$ for $\mathrm{AD}$ vs. $\mathrm{HC}$ & 91.88 \\
\hline & & & & AD vs. MCI & 78.63 \\
\hline & & & & MCI vs. HC & 90.17 \\
\hline & & & & AD vs. MCI vs. HC & 78.49 \\
\hline
\end{tabular}

$\begin{array}{ccl} & & \text { Subject: AD 16, 10 HC } \\ & {[61]} & \text { Channel: } 128 \\ 4 & \text { (2018) } & \text { Sampling rate: } 2000 \mathrm{~Hz} \\ & \text { Recording: Resting state }\end{array}$

FFT, CWT Random Forest

$83.49,85.09$ 
There is unavailability of long-term Open Access EEG Data for epilepsy. As epilepsy seizures do not occur continuously; the diagnosis of epilepsy needs long term EEG recording (more than $2-3 \mathrm{~h}$ at least). The lack of observations is one of the key explanations for the poor efficiency of prediction algorithms. The values in the observed data are almost zero, since the wearables or embedded devices with limited storage and storage space are not interconnected with each other for many potential reasons. Learning from corrupt or absent data did not take the machine learning community much into consideration. However, missing indicators must be added in models, which can provide important data to make predictions [22].

In Ref. [28], the authors use statistical models for classification, which allows seizure detection simultaneously in the different brain rhythms, complying with current medical practices. However, it has some limitations wherein firstly it is difficult to define the sliding time window and the overlap between epochs because of the very high dynamics of epileptic signals. Second, it must define regularization conditions for the training phase so that random peaks, noise and devices can be taken into account that can contribute to false positive effects. Third, seizures have variable and dynamic offsets that represent the complicated existence of the various forms of epilepsy. For instance, if brain waves slow down, it is difficult to monitor changes from seizure to seizure and classification errors are created. Although the majority of research in seizure warning systems has focused on EEG-based methods for seizure detection, currently available systems suffer from poor sensitivity and specificity [35].

Due to unnecessary extraction of features, time consumption and computing costs would increase. There is a need for quick predictions of relatively low power hardware and cheaper computer costs so that the ES prediction method can be implemented in real time. Even as DL have greatly rectified the problem of feature extraction from pre-processed data, the constraint is that these methods need a large amount of information to predict effectively [32].
Many EEG studies probably indicate that they are homogeneous in their clinical classes. If this is not so, the group variations that have been identified do not adequately represent the magnitude of the EEG deviation in individual kids with ADHD [40]. Other major issues not effectively addressed are that of comorbidity; the simultaneous presence of two or more diseases or medical conditions in a patient such as autism, which is a learning disorder falling in the ADHD spectrum. We should expect to see differing and contradictory results in such cases [40]. Different researchers are studying EEG for different age groups. Major evidence suggests that EEG abnormalities in ADHD are influenced by gender in childhood but requires further investigation [39].

\section{Conclusion}

EEG signals are used as an authentic indicator which allows observing mental states and diseases related to the brain. It is used as the analyzing tool of neurological diseases, classification and rehabilitation. The methodologies of analyzing the diseases also differ according to the diseases. Some of the techniques to analyze EEG waveform uses time-frequency preprocessing while others use focal waves to detect the active side in the EEG signal.

In this manuscript, different signal analysis methods including linear, frequency domain, time-frequency, and nonlinear techniques are discussed for the detection of neurological disorders. This paper has also explored several diagnosis techniques for three prominent neurological disorders like Alzheimer's disease, Epilepsy and ADHD along with a discussion on the limitations in the analysis of these disorders. With these details, various open sources data sets and tools are provided for further research for improving the automated prediction of neurological disorders.

\section{Disclosures}

All authors declare that there is no conflict of interests in this paper.

\section{References}

1. V. Janga, P. E. S. Reddy, "A review on Machine Learning Techniques for Neurological disorders estimation by Analyzing EEG Waves,” International Journal of Trend in Scientific Research and Development 2(1), 824-831 (2017).

2. S. Siuly, Y. Zhang, "Medical Big Data: Neurological Diseases Diagnosis Through Medical Data Analysis," Data Science and Engineering 1(2), 54-64 (2016).

3. U. Raghavendra, U. R. Acharya, and H. Adeli, "Artificial Intelligence Techniques for Automated Diagnosis of Neurological Disorders," European Neurology 82(1-3), 41-64 (2020).

4. M. McVoy, S. Lytle, E. Fulchiero, M. E. Aebi, O. Adeleye, and M. Sajatovic, "A systematic review of quantitative EEG as a possible biomarker in child psychiatric disorders," Psychiatry Research 279, 331-344 (2019).

5. D. P. Subha, P. K. Joseph, R. Acharya U, and C. M. Lim, "EEG signal analysis: a survey," Journal of Medical Systems 34(2), 195-212 (2010).

6. A. Khosla, P. Khandnor, and T. Chand, "A comparative analysis of signal processing and classification methods for different applications based on EEG signals,” Biocybernetics and Biomedical Engineering 40(2), 649-690 (2020). 
7. G. Birajadar, C. Bhyri, “Comprehensive survey on EEG analysis for detecting brain disorders,” Mukt Shabd Journal IX(VI), 2258-2262 (2020).

8. S. Siuly, Y. Li, and Y. Zhang, EEG Signal Analysis and Classification, Springer Intenational Publishing, Switzerland (2016). ISBN 978-3-319-47653-7.

9. U. Halici, E. Agi, C. Özgen, and I. Ulusoy, "Analysis and classification of EEG signals for brain computer interfaces," in International Conference on Cognitive Neuroscience X, 1-5 September, Turkey (2008).

10. M. Sheoran, S. Kumar, and S. Chawla, "Methods of denoising of electroencephalogram signal: A review," International Journal of Biomedical Engineering and Technology 18(4), 385-395 (2015).

11. X. Jiang, G. Bin Bian, and Z. Tian, "Removal of artifacts from EEG signals: A review," Sensors 19(5), 1-18 (2019).

12. M. M. N. Mannan, M. A. Kamran, and M. Y. Jeong, "Identification and removal of physiological artifacts from electroencephalogram signals: A review," IEEE Access 6, 30630-30652 (2018).

13. M. Lakshmi, D. Prasad, and D. Prakash, "Survey on EEG signal processing methods," International Journal of Advanced Research in Computer Science and Software Engineering 4(1), 84-91 (2014).

14. A. R. Mane, P. S. D. Biradar, and P. R. K. Shastri, "Review paper on Feature Extraction Methods for EEG Signal Analysis," International Journal of Emerging Trend in Engineering and Basic Sciences 2(1), 545-552 (2015).

15. M. Kazakeviciute, A. Juozapavicius, and R. Samaitiene, "Morphological filtering of EEG," Materials Physics and Mechanics 9, 185-193 (2010).

16. B. Singh, H. Wagatsuma, "A Removal of Eye Movement and Blink Artifacts from EEG Data Using Morphological Component Analysis," Computational and Mathematical Methods in Medicine 2017, 1861645 (2017).

17. O. K. Fasil, R. Rajesh, "Empirical Mode Decomposition of EEG Signals for the Effectual Classification of Seizures," Advances in Neural Signal Processing, 1-13 (2020).

18. A. Borowicz, "Using a multichannel Wiener filter to remove eye-blink artifacts from EEG data," Biomedical Signal Processing and Control 45, 246-255 (2018).

19. L. Hu, Z. Zhang, EEG signal processing and feature extraction, Springer, Singapore (2019).

20. M. Adamou, T. Fullen, and S. L. Jones, "EEG for Diagnosis of Adult ADHD: A Systematic Review With Narrative Analysis," Frontiers in Psychiatry 11, 871 (2020).

21. A. Tenev, S. Markovska-Simoska, L. Kocarev, J. Pop-Jordanov, A. Müller, and G. Candrian, "Machine learning approach for classification of ADHD adults," International Journal of Psychophysiology 93(1), 162-166 (2014).

22. K. Rasheed, A. Qayyum, J. Qadir, S. Sivathamboo, P. Kwan, L. Kuhlmann, T. O'Brien, and A. Razi, "Machine Learning for Predicting Epileptic Seizures Using EEG Signals: A Review," IEEE Reviews in Biomedical Engineering 14, 139-155 (2021).

23. M. Zuhair, S. Thomas, "Classificat ion f patient by analyzing EEG signal using DWT and least square support vector machine," Advances in Science, Technology and Engineering Systems 2(3), 1280-1289 (2017).

24. C.E. Stafstrom, L. Carmant, "Seizures and epilepsy: an overview for neuroscientists," Cold Spring Harbor Perspectives in Medicine 5(6), a022426 (2015).

25. H. U. Amin, M. Z. Yusoff, and R. F. Ahmad, "A novel approach based on wavelet analysis and arithmetic coding for automated detection and diagnosis of epileptic seizure in EEG signals using machine learning techniques," Biomedical Signal Processing and Control 56, 101707 (2020).

26. E. M. Imah, A. Widodo, “A comparative study of machine learning algorithms for epileptic seizure classification on EEG signals," in 2017 International Conference on Advanced Computer Science and Information Systems (ICACSIS), 401-407 (2017).

27. M. K. Hasan, M. A. Ahamed, M. Ahmad, and M. A. Rashid, "Prediction of Epileptic Seizure by Analysing Time Series EEG Signal Using k -NN Classifier,” Applied Bionics and Biomechanics 2017, 6848014 (2017).

28. A. Quintero-Rincón, M. Pereyra, C. D'Giano, M. Risk, and H. Batatia, "Fast statistical model-based classification of epileptic EEG signals,” Biocybernetics and Biomedical Engineering 38(4), 877-889 (2018).

29. A. Bhattacharyya, M. Sharma, and R. Bilas, "A novel approach for automated detection of focal EEG signals using empirical wavelet transform,” Neural Computing and Applications 29, 47-57 (2016).

30. T. Zhang, W. Chen, and M. Li, "Classification of inter-ictal and ictal EEGs using multi-basis MODWPT, dimensionality reduction algorithms and LS-SVM: A comparative study," Biomedical Signal Processing and Control 47, 240-251 (2019).

31. Ö. Türk, M. S. Özerdem, "Epilepsy Detection by Using Scalogram Based Convolutional Neural Network from EEG Signals,” Brain Science 9(5), 115 (2019).

32. H. Daoud, M. Bayoumi, "Deep Learning based Reliable Early Epileptic Seizure Predictor," in IEEE Biomedical Circuits and Systems Conference (BioCAS), 1-4 (2018).

33. K. Adalarasu, P. Shrisowmya, and M. Jagannath, "Identifying Pre-ictal Period of Seizure and Better Brain Lobes for Seizure Detection using EEG Biomarkers Identifying Pre-ictal Period of Seizure and Better Brain Lobes for Seizure Detection using EEG Biomarkers," Journal of Physics: Conference Series 1451, 012010 (2020).

34. Z. Gao, G. Lu, P. Yan, C. Lyu, X. Li, and W. Shang, "Automatic Change Detection for Real-Time Monitoring of EEG Signals,” Frontiers in Physiology 9, 325 (2018). 
35. S. Ramgopal, S. Thome-Souza, M. Jackson, N. E. Kadish, I. S. Fernández, J. Klehm, W. Bosl, C. Reinsberger, S. Schachter, and T. Loddenkemper, "Seizure detection, seizure prediction, and closed-loop warning systems in epilepsy," Epilepsy \& Behavior 37, 291-307 (2014).

36. EEG Data Download (accessed 14 July 2021). [https://www.ukbonn.org/epileptologie/ag-lehnertz-downloads/].

37. Datasets - DTIC-MdM Strategic Program: Data-Driven Knowledge Extraction (UPF) (accessed 14 July 2021 ). [https://www.upf.edu/web/mdmdtic/datasets].

38. "CHB-MIT Scalp EEG Database v1.0.0," (accessed 14 July 2021). [https://physionet.org/content/chbmit/1.0.0/].

39. M.-Y. Chang, C.-S. Ouyang, C.-T. Chiang, R.-C. Yang, R.-C. Wu, H.-C. Wu, and L.-C. Lin, "A New Method of Diagnosing Attention-Deficit Hyperactivity Disorder in Male Patients by Quantitative EEG Analysis," Clinical EEG and Neuroscience 50(5), 339-347 (2019).

40. R. J. Barry, A. R. Clarke, and S. J. Johnstone, “A review of electrophysiology in attention-deficit/hyperactivity disorder: I. Qualitative and quantitative electroencephalography," Clinical Neurophysiology 114(2), 171-183 (2003).

41. H. Chen, W. Chen, Y. Song, L. Sun, and X. Li, "EEG characteristics of children with attention-deficit/hyperactivity disorder," Neuroscience 406, 444-456 (2019).

42. J. J. Newson, T. C. Thiagarajan, "EEG Frequency Bands in Psychiatric Disorders: A Review of Resting State Studies," Frontiers in Human Neuroscience 12, 521 (2019).

43. S. M, N. G. Cholli, and S. Nayak, "Classification of Attention Deficit Hyperactivity Disorder (ADHD) Considering Diagnosis and Treatment," International Journal of Modern Education and Computer Science 11(6), 26-42 (2019).

44. R. Gabriel, M. M. Spendola, A. Mesquita, and A. Z. Neto, "Identification of ADHD cognitive pattern disturbances using EEG and wavelets analysis," in IEEE $17^{\text {th }}$ International Conference on Bioinformatics and Bioengineering (BIBE), 157-162 (2017).

45. F. Fahimi, C. Guan, W. B. Goh, K. K. Ang, C. G. Lim, and T. S. Lee, "Personalized features for attention detection in children with Attention Deficit Hyperactivity Disorder," in $39^{\text {th }}$ Annual International Conference of the IEEE Engineering in Medicine and Biology Society (EMBC), 414-417 (2017).

46. A. Kamida, K. Shimabayashi, M. Oguri, T. Takamori, N. Ueda, Y. Koyanagi, N. Sannomiya, H. Nagira, S. Ikunishi, Y. Hattori, K. Sato, C. Fukuda, Y. Hirooka, and Y. Maegaki, "EEG power spectrum analysis in children with ADHD," Yonago Acta Medica 59(2), 169-173 (2016).

47. M. R. Mohammadi, A. Khaleghi, A. M. Nasrabadi, S. Rafieivand, M. Begol, and H. Zarafshan, "EEG classification of ADHD and normal children using non-linear features and neural network," Biomedical Engineering Letters 6(2), 66-73 (2016).

48. M. Rezaeezadeh, S. Shamekhi, and M. Shamsi, “Attention Deficit Hyperactivity Disorder Diagnosis using non-linear univariate and multivariate EEG measurements: a preliminary study," Physical and Engineering Sciences in Medicine 43(2), 577-592 (2020).

49. M. Altınkaynak, N. Dolu, A. Güven, F. Pektaş, S. Özmen, E. Demirci, and M. İzzetoğlu, "Diagnosis of Attention Deficit Hyperactivity Disorder with combined time and frequency features," Biocybernetics and Biomedical Engineering 40(3), 927-937 (2020).

50. H. Chen, Y. Song, and X. Li, “A deep learning framework for identifying children with ADHD using an EEG-based brain network," Neurocomputing 356, 83-96 (2019).

51. A. Alex, S. Coelli, A. M. Bianchi, L. Ponzini, E. Buzzi, and M. P. Canevini, "EEG analysis of brain activity in attention deficit hyperactivity disorder during an attention task," in IEEE $3^{\text {rd }}$ International Forum on Research and Technologies for Society and Industry (RTSI), 1-4 (2017).

52. A. E. Alchalabi, S. Shirmohammadi, A. N. Eddin, and M. Elsharnouby, "FOCUS: Detecting ADHD patients by an EEGbased serious game," IEEE Transactions on Instrumentation and Measurement 67(7), 1512-1520 (2018).

53. C. Lynch, "World Alzheimer Report 2019: Attitudes to dementia, a global survey," Alzheimer's Dementia 16(S10), e038255 (2020).

54. M. Tanveer, B. Richhariya, R. U. Khan, A. H. Rashid, P. Khanna, M. Prasad, and C. T. Lin, "Machine learning techniques for the diagnosis of alzheimer's disease: A review," ACM Transactions on Multimedia Computing, Communications, and Applications 16(1s), 30 (2020).

55. S. J. Ruiz-Gómez, C. Gómez, J. Poza, G. C. Gutiérrez-Tobal, M. A. Tola-Arribas, M. Cano, and R. Hornero, "Automated multiclass classification of spontaneous EEG activity in Alzheimer's disease and mild cognitive impairment," Entropy 20(1), 1-15 (2018).

56. F. J. Fraga, G. Q. Mamani, E. Johns, G. Tavares, T. H. Falk, and N. A. Phillips, "Early diagnosis of mild cognitive impairment and Alzheimer's with event-related potentials and event-related desynchronization in N-back working memory tasks," Computer Methods and Programs in Biomedicine 164, 1-13 (2018).

57. L. Tylová, J. Kukal, V. Hubata-Vacek, and O. Vyšata, "Unbiased estimation of permutation entropy in EEG analysis for Alzheimer's disease classification," Biomedical Signal Processing and Control 39, 424-430 (2018).

58. L. D. Deepthi, D. Shanthi, and M. Buvana, “An intelligent Alzheimer's disease prediction using convolutional neural network (CNN), " International Journal of Advanced Research in Engineering and Technology 11(4), 12-22 (2020).

59. N. N. Kulkarni, V. K. Bairagi, “Extracting Salient Features for EEG-based Diagnosis of Alzheimer's Disease Using Support Vector Machine Classifier,” IETE Journal of Research 63(1), 11-22 (2017). 
60. C. Ieracitano, N. Mammone, A. Bramanti, A. Hussain, and F. C. Morabito, "A Convolutional Neural Network approach for classification of dementia stages based on 2D-spectral representation of EEG recordings," Neurocomputing 323, 96-107 (2019).

61. P. Durongbhan, Y. Zhao, L. Chen, P. Zis, M. D. Marco, Z. C. Unwin, A. Venneri, X. He, S. Li, Y. Zhao, D. J. Blackburn, and P. G. Sarrigiannis, "A dementia classification framework using frequency and time-frequency features based on EEG signals," IEEE Transactions on Neural Systems and Rehabilitation Engineering 27(5), 826835 (2019).

62. N. Houmani, F. Vialatte, E. Gallego-Jutglà, G. Dreyfus, V.-H. Nguyen-Michel, J. Mariani, and K. Kinugawa, "Diagnosis of Alzheimer's disease with electroencephalography in a differential framework," PLoS One 13(3), 1-19 (2018). 


\section{Appendix A}

\author{
AD: Alzheimer's disease \\ ADHD: Attention-deficit/hyperactivity disorder \\ ANOVA: Analysis of variance \\ ApEn: Approximate entropy \\ Bi-LSTM: Bidirectional Long short-term memory \\ BSS: Blind Source Separation \\ CNN: Convolutional Neural Network \\ CTM: Central tendency measure \\ CWT: Continuous Wavelet Transform \\ DCNN: Deep Convolutional Neural Network \\ DispEn: Dispersion entropy \\ DSM: Diagnostic and Statistical Manuel of Mental \\ Disorders \\ DWT: Discrete Wavelet Transformation \\ ECG: Electrocardiogram \\ EEG: Electroencephalogram \\ EMD: Empirical Mode Decomposition \\ EMG: Electromyography \\ EOG: Electrooculogram \\ ERP: Event-related potentials \\ EWT: Empirical wavelet transform \\ fMRI: Functional Magnetic Resonance Imaging \\ GGD: Generalized Gaussian distribution \\ GRIVQ: Generalized relevance learning vector \\ quantization \\ HC: Healthy control \\ ICA: Independent Component Analysis \\ ICD: International Classification for Diseases \\ iEEG: Invasive electroencephalography \\ IMFs: Intrinsic Mode Functions
}

KNN: K-Nearest Neighbor

LDA: Linear Discriminant Analysis

LS-SVM: Least-Squares Support Vector Machine

LSTM: Long short-term memory

MCA: Morphological component analysis

MCI: Mild Cognitive Impairment

MEG: Magnetoencephalogram

MLP: Multi-layer Perceptron Neural Network

MODWPT: Maximal over- lap discrete wavelet package transform

mvSE: Multivariate Sample Entropy

NIRS: Near Infrared Spectroscopy

PCA: Principal Component Analysis

PET: Positron Emission Tomography

PSD: Power spectral density

QDA: Quadratic Discriminant Analysis

RBF: Radial basis function

RK-LS-SVM: LS-SVM with RBF kernel

rsEEG: Resting-state EEG

SampEn: Sample Entropy

SNR: Signal Noise Ratio

SOP: Seizure Occurrence Period

SPH: Seizure Prediction Horizon

STFT: Short-time Fourier Transform

SVM: Support Vector Machine

TBAR: Theta/ (alpha + beta) ratio

TBR: Theta/beta ratio

TFA: Time-frequency Analysis

WHO: World Health Organizations

WT: Wavelet transform 